\title{
Versatile Encapsulation and Synthesis of Potent Therapeutic Liposomes by Thermal Equilibration
}

\author{
Steven A. Roberts ${ }^{a}$, Chaebin Lee ${ }^{b, \neq}$, Shrishti Singha, ${ }^{a,}$, and Nitin Agrawal ${ }^{\text {* }}$ \\ aGeorge Mason University, 4400 University Drive, MS 2A1, Fairfax, VA, 22033 USA. \\ ${ }^{b}$ Center for Cancer and Immunology Research, Children's National Hospital, Washington, DC. \\ ${ }^{*}$ Corresponding Author \\ ${ }^{\sharp}$ Equal Contribution
}

Keywords: drug delivery, liposome, encapsulation, molecular dynamics

The wide-scale use of liposomal delivery systems is hampered by difficulties in obtaining potent liposomal suspensions. Passive and active loading strategies have been proposed to formulate drug encapsulated liposomes, but are limited by low efficiencies (passive) or high drug specificities (active). Here, we present an efficient and universal loading strategy for synthesizing therapeutic liposomes. Integrating a thermal equilibration technique with our unique liposome synthesis approach, co-loaded targeting liposomes can be engineered in an efficient and scalable manner with potencies 200-fold higher than typical passive encapsulation techniques. We demonstrate this capability through simultaneous co-loading of hydrophilic and hydrophobic small molecules and through targeted delivery of liposomal Doxorubicin to a metastatic breast cancer cell line MDA-MB-231. Molecular dynamic simulations are used to explain interactions between Doxorubicin and liposome membrane during thermal equilibration. By addressing the existing challenges, we have developed an unparalleled approach that will facilitate the formulation of novel theranostic and pharmaceutical strategies. 


\section{Introduction}

Decades of research have resulted in a variety of liposomal formulations for use in both therapeutic ${ }^{1-3}$ and theranostic ${ }^{4-6}$ applications. Advances in synthesis procedure and membrane functionalization have led to targeting and stealth capabilities, which can increase bioavailability at disease sites several fold with minimal side effects, compared to systemically administered drugs ${ }^{7,8}$. These improvements have in turn led to the FDA approval and commercial use of several liposomal drugs, including Doxil ${ }^{\circledR}$ for cancer $^{9}$, Abelcet ${ }^{\circledR}$ for fungal infections ${ }^{10}$, and DepoDur ${ }^{\circledR}$ for pain management ${ }^{11}$, and more recent Moderna/Pfizer vaccines for Covid-1912. While substantial progress has been made in the development of novel liposomal formulations, considerably fewer strategies to increase the potency and concentration of therapeutics within the vehicles have been developed.

Sequestration of therapeutic compounds has traditionally been accomplished by either passive or active encapsulation approaches. The passive approach can sequester molecules irrespective of their polarity ${ }^{13}$, where lipophilic molecules reside within the lipid bilayer membrane and hydrophilic molecules are contained within the core. During vesiculation, liposomes form within the media containing drug, encapsulating the drug in the process. This approach is typically done with dilute solutions, and therefore the encapsulation efficiencies are relatively low ${ }^{14}$. This leaves much of the drug to be lost during secondary purification steps. Furthermore, organic solvents (e.g. chloroform and ethanol) are typically used, that can inactivate certain molecules ${ }^{15}$.

Active loading techniques have been developed that drastically improve encapsulation efficiencies and minimize the amount of non-encapsulated drug. The sequestration is driven by transmembrane pH or ion gradients that cause the influx of hydrophilic molecules to the core of liposomes ${ }^{16-18}$. This approach has been successfully utilized to sequester weakly basic drugs with encapsulation efficiencies of nearly $100 \%$. Once inside the liposome, drug molecules precipitate out of the solution, increasing their retention time. While this technique overcomes many of the barriers to passive encapsulation, it is limited to the molecules that can pass through the membrane. Furthermore, decorating the membrane with targeting moieties may require a second step to remove any unbound targeting molecules that may result in the loss of encapsulated drugs.

We have previously demonstrated a simple approach for producing purified drug loaded liposomes ${ }^{19}$. Using this method, we rapidly synthesize concentrated populations of small unilamellar liposomes (SULs) with a very narrow polydispersity index (typically near 0.13 ). The approach relies on passive encapsulation to sequester small molecules, and as such, the encapsulation efficiency is relatively low. Therefore, a technique that can enhance the encapsulation efficiency utilizing small amount of compounds is desired. Here, we demonstrate a new approach for creating drug-loaded targeting liposomes using thermal equilibration, where drug molecules are sequestered at high concentrations via passive diffusion at the membrane transition temperature $\left(T_{m}\right)$. We demonstrate the universal and translational appeal of this approach by simultaneously co-loading both hydrophilic and lipophilic small molecules. We further demonstrate the therapeutic potential of this technique by encapsulating Doxorubicin (DXR), without utilizing ion gradients typically required for active methods. Bioactivity of these liposomes was observed by actively targeting cancer cells in vitro. Finally, using molecular dynamic simulations, we explain the thermodynamics that underlie the encapsulation and model the effects of temperature on structural properties of the liposome membrane and DXR diffusion. The thermal equilibration approach presented here is a powerful universal strategy for rapidly encapsulating small molecule drugs. It offers significantly 
greater encapsulation capabilities than conventional passive encapsulation techniques and represents a feasible solution to producing therapeutic grade liposomes in a scalable manner.

\section{Experimental}

\section{$\underline{\text { Materials }}$}

Cholesterol, 1,2-Distearoyl-sn-glycero-3-phosphocholine (DSPC), 5,5'-Dithiobis(2-nitrobenzoic acid) (Ellan's reagent), L-Cysteine, and all fluorescent dyes were purchased from Sigma Aldrich (St. Louis, MO). 1,2-distearoyl-sn-glycero-3-phosphoethanolamine- $\mathrm{N}$-[maleimide(polyethylene glycol)-2000] (ammonium salt) (DSPE-PEG-Mal) was purchased from Avanti Polar lipids. Isopropyl Alcohol (IPA, 99\% Pure), Ethanol (EtOH, 99\% pure), Dimethyl Sulfoxide (DMSO), desalting columns, dye removal columns, and Trypsin-EDTA were purchased from Fisher Scientific (Hampton, NH). Glass syringes were purchased from Hamilton (Reno, NV) and luer lock dispensing needles were purchased from Jensen Global (Santa Barbara, CA). Amicon Ultra $100 \mathrm{kDa}$ centrifugal filter units were purchased from EMD-Millipore (Billerica, MA). A NanoJ et syringe pump from Chemyx Inc. (Stafford, TX) was used for all experiments. Dulbecco's Modified Eagle Medium (DMEM), Fetal Bovine Serum (FBS), and Penicillin-Streptomycin antibiotic were purchased from VWR (Radnor, PA). Doxorubicin (DXR) and WST-1 proliferation assay were purchased from Cayman Chemical (Ann Arbor, Michigan). MDA-MB-231 metastatic breast cancer cell line was acquired from ATCC (Manassas, VA).

\section{Synthesis of Small Unilamellar Liposomes}

Liposomes were synthesized as previously described ${ }^{19}$. Briefly, DSPC and Cholesterol were dissolved in isopropyl alcohol at a constant 2:1 molar ratio to a final concentration of $10 \mathrm{mM}$ and $5 \mathrm{mM}$, respectively. The alcoholic solution was injected using a syringe pump at $100 \mu \mathrm{L} \cdot \mathrm{min}^{-1}$ into a glass vial containing distilled water preheated at $55^{\circ} \mathrm{C}$ to obtain a homogeneous population of liposomes. Typical synthesis volumes would be a total of $10 \mathrm{~mL}$ ( $1 \mathrm{~mL}$ alcoholic lipid suspension to $9 \mathrm{~mL}$ warm distilled water). The aqueous solution was constantly stirred at $600 \mathrm{rpm}$ during infusion, and stirring was continued for 3 minutes following infusion. After the liposomal suspension was cooled down to room temperature with continuous stirring, liposomes were concentrated 20 fold to a volume of $500 \mu \mathrm{L}$ and a final lipid concentration of $20 \mathrm{mM}$ using filter centrifugation $(100 \mathrm{kDa}, 6000 \mathrm{xg})$ at $4^{\circ} \mathrm{C}$ for 30 minutes. The particles were stored at $4^{\circ} \mathrm{C}$ until use.

\section{Antibody Coupling}

Antibody coupling was accomplished through the addition of functionalized PEGylated lipids (DSPE-PEGMal). DSPE-PEG-Mal was dissolved to a concentration of $25 \mathrm{mg} / \mathrm{mL}$ in DMSO and added to the liposomal suspension containing small molecules (e.g. DXR, Nile Red, or Fluorescein) to a final molar ratio of $1 \%$ (DSPE/DSPC). The suspension was incubated for one hour at $55^{\circ} \mathrm{C}$ to increase fluidity of the membrane. Simultaneously, antibodies targeting CD44 were thiolated by incubation with Traut's reagent at a ratio of 3:1 (protein: Traut) for 1 hour. Unreacted Traut's reagent was removed by passing antibody suspensions through a desalting column and the resulting thiolated antibody was added to the liposomal suspension at a molar ratio of 1:1 (DSPE:Protein). The coupling reaction occurred overnight at $4{ }^{\circ} \mathrm{C}$. Finally, nonreacted maleimide groups were neutralized by a 15 minute incubation with $\beta$-mercaptoethanol at a molar concentration of 3:1 ( $\beta$-mercaptoethanol:DSPE). The resulting suspension was then filtered as previously described to remove all non-encapsulated and unreacted molecules. 


\section{Quantification of PEG-maleimide and Coupling Efficiency on the Liposomes}

The amount of DSPE-PEG-maleimide on liposomes was evaluated following Ellman's test ${ }^{20}$. Briefly, $120 \mu \mathrm{L}$ of the functionalized liposomes were mixed with $30 \mu \mathrm{L}$ of L-Cysteine $(0.36 \mathrm{mM})$ using a thermomixer at $700 \mathrm{rpm}$ for 2 hours at room temperature. The particles were removed by filter centrifugation for 30 minutes at $6000 \mathrm{xg}$. In a trans parent 96 -well plate, $100 \mu \mathrm{L}$ of the supernatant and $50 \mu \mathrm{L}$ of 5, 5'-Dithiobis (2nitrobenzoic acid) $(0.144 \mathrm{mM})$ were added and mixed for 30 minutes at room temperature. The absorbance at $409 \mathrm{~nm}$ was measured using a microplate reader, and the unreacted L-Cysteine was determined using a standard curve. The amount of conjugated DSPE-PEG-maleimide was calculated by subtracting unreacted L-Cysteine from the total amount of L-Cysteine added to the functionalized liposomes, and the DSPE-PEG-MAL to DSPC ratio was evaluated.

Thermal Equilibration for Small Molecule Encapsulation

In this work, a hydrophilic (Fluorescein sodium salt) and two hydrophobic compounds (Nile red and Doxorubicin) were selected for encapsulation using thermal equilibration. Fluorescein sodium salt was prepared in water at a concentration of $1.5 \mathrm{M}$ while Nile Red and Doxorubicin $\mathrm{HCl}$ were prepared in DMSO at concentrations of $30 \mathrm{mM}$ and $172 \mathrm{mM}$, respectively. The concentrations of small molecule are detailed in the results section. The small molecule solution was added to liposome suspension and incubated at desired temperatures and times. After liposomes were cooled to $4{ }^{\circ} \mathrm{C}$, non-encapsulated DXR was removed from suspension using a Zeba Spin desalting column with a $7 \mathrm{kDa}$ cutoff, while excess fluorescent dyes were removed using dye removal columns. For targeted liposomes, DSPE-PEG-Mal was dissolved to a concentration of $25 \mathrm{mg} / \mathrm{mL}$ in DMSO and added to a final mol ratio of $1 \%$ (DSPE/DSPC).

\section{Analysis of Small Molecule Encapsulation and Release}

Encapsulated compound concentrations were analyzed using a Thermo Scientific Varioskan Flash multimode reader. DXR concentration was quantified using an excitation of $525 \mathrm{~nm}$ and an emission of $580 \mathrm{~nm}$. Liposomes were solubilized in $0.1 \%$ Triton $x-100$ before measuring the fluorescence. The fluorescence intensity was compared to the standard curve which was prepared by measuring the fluorescence of DXR in $0.1 \%$ Triton $x-100$. To quantify the concentration of co-loaded samples, after dissolving liposomes in $0.1 \%$ Triton $x-100$, the fluorescence was measured at an excitation of $488 \mathrm{~nm}$ and emission of $512 \mathrm{~nm}$ in water for fluorescein. The samples were then desiccated for several hours under vacuum and redissolved in IPA. The fluorescence of Nile Red was measured using an excitation of $550 \mathrm{~nm}$ and an emission of $625 \mathrm{~nm}$. The fluorescence intensities of fluorescein and Nile Red from the sample were compared to standard curves prepared with either triton x-100 in water (fluorescein) or IPA (Nile Red) to evaluate encapsulated compound concentrations.

DXR release was investigated by diluting samples 20 -fold in PBS with 10\% FBS and incubating for up to 48 hours at $37^{\circ} \mathrm{C}$. At indicated time points, liposome suspension was purified by multiple filtrations to remove released DXR. First, the liposome suspension was filtered through a $7 \mathrm{kDa}$ desalting column to remove free doxorubicin followed by another filtration using a $100 \mathrm{kDa}$ Amicon ultra centrifugal filter unit to remove any serum bound DXR. Liposomes were dissolved in $0.1 \%$ Triton $x-100$, and the fluorescence of each sample was measured using an excitation of $525 \mathrm{~nm}$ and an emission of $580 \mathrm{~nm}$. The remaining DXR in liposomes was calculated by comparing the fluorescence intensities to a standard curve prepared with DXR in $0.1 \%$ triton $x-100$. 


\section{Stability of Liposomes in Serum}

The effect of serum on liposome stability was tested by monitoring the particle size change in either $\mathrm{H} 2 \mathrm{O}$, PBS, or PBS with $10 \%$ FBS for up to 24 hours at $37^{\circ} \mathrm{C}$. Briefly, $25 \mu \mathrm{L}$ of liposome solution was dispersed into either $\mathrm{H} 2 \mathrm{O}, \mathrm{PBS}$, or PBS with $10 \% \mathrm{FBS}$ and mixed by thermomixer at $37^{\circ} \mathrm{C}$. Samples were collected at different time points $(0,1,4,16$, and 24 hours $)$, and $20 \mu \mathrm{L}$ of each sample was introduced into $500 \mu \mathrm{L}$ of fresh $\mathrm{H} 2 \mathrm{O}$ for size measurement using DLS.

\section{Quantification of Phospholipid Concentration}

Phospholipid concentration was empirically determined via Stewart Assay ${ }^{21}$. Briefly, liposomal suspensions were dehydrated at $70{ }^{\circ} \mathrm{C}$ in polypropylene microcentrifuge tubes. The lipids were then dissolved in 500uL chloroform, and an equal volume of Stewart's reagent (100 mM FeCl 3 and $400 \mathrm{mM}$ $\mathrm{NH}_{4} \mathrm{SCN}$ in DI water) was added and vortexed for 20 seconds. The tubes were centrifuged for 10 minutes at $1000 \times \mathrm{g}$ to create a phase separation. The chloroform layer was then analyzed in a quartz cuvette for its absorbance at $472 \mathrm{~nm}$ and compared to a standard curve to determine the phospholipid concentration. The standard curve was made using a serial dilution of lipids in cholesterol at a 2:1 ratio.

\section{Cell Culture and Therapeutic Delivery}

MDA-MB-231 cells were grown in DMEM containing 10\% FBS and 1\% Penicillin-Streptomycin solution. The cells were cultured at $70 \%$ confluence and plated in a 96 well plate at a density of 5000 cells/well. The cells were incubated undisturbed for two days prior to any experimentation. Liposomal suspensions were UV sterilized prior to introduction to cell cultures. On the second day, the liposomal carriers containing DXR were introduced to the cultures at the $I_{50}$ of DXR $\left(0.01 \mu \mathrm{M}^{22}\right)$. Proliferating cells were quantified and normalized by the control using WST-1 proliferation assay following the manufactural protocol.

\section{Membrane Simulations}

Simulations are performed on a section of the lipid membrane consisting of DSPC and cholesterol held in a 2:1 molar ratio. The membrane section was constructed using Charmm-27 GUI Membrane Builder ${ }^{23}$, and designed to consist of 256 total lipid molecules and 128 cholesterol molecules. The membrane was hydrated using 30 water molecules per lipid. Nanoscale Molecular Dynamics (NAMD) was used to thermally equilibrate the bilayer system for $1 \mathrm{~ns}$ at designated temperatures. Production runs were also conducted at designated temperatures with a time step of 1 femtosecond and data collection every 20 femtoseconds. Every simulation was run for at least 100 ns, yielding at least 5000 data points per temperature. Trajectories of the simulations were visualized using Visual Molecular Dynamics (VMD). Further information on the simulation setup is available in the supplementary information.

\section{Statistical Data Analysis}

Statistical analysis was conducted using GraphPad Prism 5 (La Jolla, CA). For each condition, at least three samples were independently prepared, and each sample was analyzed three times. Statistical analysis was performed using either a student's unpaired t-test or a One-Way Analysis of Variance (ANOVA) with a Tukey post-test. Data was deemed statistically significant if $p$ values were less than 0.05 . Graphs show the mean and the standard error of the mean (SEM) of sample groups.

\section{Results}




\section{Synthesis Approach and Maximum Equilibration Calculation}

Typical liposome synthesis techniques ${ }^{15,24,25}$ require extensive secondary processing (e.g. sonication ${ }^{26}$, extrusion ${ }^{27}$ freeze thawing ${ }^{28}$ ) to generate monodisperse SULs. An alternative method that has been utilized to generate SUL's without extensive post processing is through the injection method (Figure 1a). The injection method is advantageous in its simplicity but typically yields low encapsulation efficiencies ${ }^{19}$. To address this, we have developed a novel approach to efficiently and rapidly sequester small molecules.

\section{Liposome Purification and Concentration}

Small unilamellar liposomes are difficult to concentrate without losing part of the population in the process due to their low densities ${ }^{29,30}$. Therefore, we proposed to use filter centrifugation instead of ultracentrifugation. To compare the efficiency of ultracentrifugation and filter centrifugation, raw liposome without loading any compound were tested. Empty liposomes were homogeneous with a polydispersity of less than 0.2 . By filter centrifuging for thirty minutes at $6000 \times \mathrm{g}$, we effectively concentrated the liposomes 20 -fold while retaining the characteristic size and lipid concentration (Figure $\mathbf{1 b}$ and $\mathbf{1 c}$ ). The mean population diameter of liposomes increased from $86.6 \mathrm{~nm}( \pm 4.5 \mathrm{~nm})$ following synthesis to $135.3 \mathrm{~nm}( \pm 7.7 \mathrm{~nm})$ following ultracentrifugation at $60000 \mathrm{x}$ g for two hours. Furthermore, by analyzing the lipid concentration, we found that only $42 \%$ ( $\pm 2.1 \%$ ) of the lipids were retained, suggesting that ultracentrifugation can only pellet larger liposomes within the population. As further proof of this, we analyzed supernatant of the ultracentrifuged sample using DLS and routinely found liposomes with diameters of $80 \mathrm{~nm}$ (data not shown). Notably, there was a non-significant change in liposome diameter following filter centrifugation $(85.3 \pm 4.3 \mathrm{~nm})$, and $79.2 \%( \pm 1.3 \%)$ of the lipids were retained. While increased centrifugation speeds $(>60,000 \times \mathrm{g})$ and longer centrifugation durations using a specialized ultracentrifugation system may improve liposome retention, the proposed filter centrifugation approach is significantly more efficient and accessible.

\section{Measurement and Quantification of Drug Encapsulation}

We evaluated the maximum equilibration concentration $\left(C_{\max }\right)$, where the number of drug molecules within the liposomes $\left(C_{E}\right)$ in solution will be proportional to the number of drug molecules in the surrounding media $\left(C_{T}\right)$. The constant of proportionality $(\alpha)$ here is the ratio of the respective volumes, where $V_{\text {Excluded }}$ is the cumulative core volume of all liposomes and $V_{\text {Total }}$ is the total volume of the solution.

$$
\begin{gathered}
C_{\text {max }}=\alpha \cdot C_{E} \\
\alpha=\frac{V_{\text {Excluded }}}{V_{\text {Total }}} \\
C_{T} \geq \alpha \cdot C_{E}
\end{gathered}
$$

$V_{\text {Excluded }}$ can be estimated by determining the inner volume of one liposome and extrapolating for the entire liposome population within the sample. Furthermore, if the drug is lipophilic, $V_{\text {Excluded }}$ can be substituted for the volume of the lipid membrane. Using our maximum equilibration model, we can predict the $D: L$ ratio as a linear function of concentration and liposome size (Figure $1 \mathbf{d}$ ). This function is linear due to the constant lipid molarity, meaning as the liposome size grows, it utilizes lipids that could otherwise be used to form additional liposomes. Additionally, if the density of the lipid membrane does not change as liposome size changes, the maximum equilibration of lipophilic drugs is solely dependent 
on $\mathrm{C}_{\mathrm{T}}$. Therefore, when equilibrating liposomes with a narrow size distribution, we can use $\mathrm{C}_{\max }$ to hypothesize the liposome potency based on extra-liposomal drug concentration (dependent on drug solubility) as well as the number of liposomes present. By concentrating the samples using filter centrifugation, we can increase the $\mathrm{C}_{\max }$ and therefore the potency of liposomes.

\section{Stability of Liposomes during Thermal Equilibration}

Several factors can affect the diffusion of small molecules across the membrane, including temperature of the system and solvents used. Alcohols such as isopropanol and ethanol offer convenient methods to increase the solubility of many drugs, however, these solvents can also deactivate or precipitate some drugs. DMSO is an alternative that is widely used to enhance the solubility of nonpolar chemicals for drug delivery. To investigate the effect of organic solvent on liposomal stability particularly at temperatures near $T_{m}$, we individually incubated aqueous solutions of isopropanol, ethanol, and DMSO with concentrated liposomal slurries at $55^{\circ} \mathrm{C}$ and analyzed their size change. We found that $10 \%$ DMSO had the least effect on liposome size (Figure 2a), with an average shift from $104 \mathrm{~nm}( \pm 0.35 \mathrm{~nm}$ ) to $107 \mathrm{~nm}$ ( \pm $0.55 \mathrm{~nm}$ ). Membrane components immediately precipitated out of the solution when introduced to alcohol concentrations of greater than $40 \%$. Samples that include $10 \%$ DMSO were stable for at least 24 hours at $55{ }^{\circ} \mathrm{C}$ (Figure $\mathbf{2 b}$ ). In all cases, we found that the stability of liposomes diminishes rapidly when exposed to temperatures above $\mathrm{T}_{\mathrm{m}}$.

\section{Encapsulation and Delivery of Chemotherapeutics from Equilibrated Liposomes}

\section{Equilibrating Liposomes with Doxorubicin}

After characterizing the stability of liposomes during thermal equilibration, we evaluated the liposomal sequestration of therapeutic compounds. Thermal equilibration (Figure 3a) relies on the incubation of concentrated liposomal solutions, where the ratio of drug:lipid (D:L) can be optimized to enhance the potency of the vehicle without using large amounts of therapeutic compounds. Liposomes were incubated with DXR being $10 \%$ of the final concentration ( $D: L$ ratios of liposomal suspension were at $1.72: 1$ ). To validate our hypothesis of temperature affecting thermal equilibration, liposomal slurries were incubated with DXR at varying temperatures ranging from 4 to $55{ }^{\circ} \mathrm{C}$ (Figure $3 \mathbf{b}$ ). We also compared this approach with a nother commonly used approach of passive encapsulation ${ }^{14}$. When held at identical initial D:L levels, equilibration at $T_{m}$ produced nearly 200 times higher $D: L$ ratios compared to passive encapsulation (0.000239 and 0.041, respectively). While this is lower than current active forms of doxorubicin sequestration, which can achieve $D: L$ of nearly 0.3 , it is also less prohibitive and can be applicable to a larger library of molecules. Interestingly, we found that a significant amount of DXR had associated with liposomes equilibrated below $T_{m}(D: L$ ratio of 0.011$)$, which we believe may be due to advantageous binding. We observed a clear exponential trend with time during the initial one-hour incubation (Figure 3c). We found no difference in liposome diameter following thermal equilibration with DXR solubilized in DMSO (Supplementary Figure 1).

A hallmark of drug delivery systems such as liposomes is their stability and slow release of therapeutics under physiological conditions. This is especially important for the delivery of chemotherapeutic compounds such as DXR which can have severe toxic side effects ${ }^{31}$. Furthermore, extravasation into thick tumors may require $24-48$ hours $^{32-34}$, and therefore formulations that release compounds too quickly are not capable of reaching the required therapeutic index upon reaching the target site. Furthermore, interaction with serum is known to induce liposome degradation. We found that the liposomes remain 
stable in water and PBS without change in size at least for 24 hours at $37^{\circ} \mathrm{C}$, whereas an increase in size of approximately $10 \%$ from $105.9 \pm 10.2 \mathrm{~nm}$ to $117.8 \pm 15.3 \mathrm{~nm}$ was observed when incubated with $10 \%$ FBS. The stability of thermally equilibrated liposomes under $10 \%$ FBS condition was confirmed by slow release of DXR with $75 \%$ of the encapsulated compound being retained after 48 hours (Figure $3 d$, Supplementary Figure 2).

\section{Delivery and Cytotoxicity of Doxorubicin Liposomes}

A distinguishing characteristic of drug delivery systems is the capability to provide therapeutic benefits through cellular uptake. To demonstrate this, we created drug loaded liposomes using the thermal equilibration technique. Liposomes were incubated with DXR (17.2mM) and DSPE-PEG-MAL (1 mol\%) for 1 hour ${ }^{35,36}$ at $55^{\circ} \mathrm{C}$ followed by purification using spin columns. However, it is likely that part of DSPE-PEGMAL will remain free, forming micelles that would be difficult to filter and subsequently interfere with antibody coupling. Therefore, retention of DSPE-PEG-MAL was calculated by determining the ratio of DSPE-PEG-MAL to DSPC lipid by Ellman's test (Supplementary Figure 3). The DSPE-PEG-MAL to DSPC ratio of $0.009 \pm 0.001$ was observed, as compared to the starting ratio of 0.01 based on 1 mol\% concentration suggesting that $>90 \%$ of DSPE-PEG-MAL was retained within the solution following the insertion protocol. The subsequent CD44 conjugation and cancer cell targeting with fluorescein loaded liposomes displayed a strong signal of the liposomal uptake further validating that neither the DSPE-PEG-MAL molecules nor the CD44 antibodies were lost as micelles or during filtration (Figure $5 \mathrm{c}$ ).

CD44 is highly expressed on MDA-MB-231 cells ${ }^{37}$ and was thus used to target DSPE-PEG-MAL incorporated liposomes after functionalization with the thiolated anti-CD44 to the maleimide group. Targeting liposomal DXR (LDXR) was incubated with MDA-MB-231 (at IC $C_{50}$ ) for 8 or 24 hours prior to being washed away with PBS. To ensure DMSO did not play a role in cell death, vehicles were generated by incubating pre-formed liposomes with DMSO in the absence of DXR. These liposomes were also functionalized with anti-CD44 IgG (HCAM-L). Free DXR was used as a positive control. Cell viability was analyzed over 72 hours using WST-1 as an indicator of proliferation and normalized to the media only control (Figure 3e). Cells demonstrated a time dependent decline in proliferation, as expected, when incubated with any form of DXR. Both 8 and 24 hours LDXR exposures eventually ended with nearly equivalent ( $p=0.173$ at 48 hours and $p=0.603$ at 72 hours, $n>3$ ) viabilities.

\section{Atomic Level Simulation of Membrane Dynamics}

Based on the predicted maximum equilibration ratios, liposomes with an average size of $100 \mathrm{~nm}$, should have $D$ : L ratios of 0.4 (Figure 1d). The prediction assumes that the inner core of the liposome contains the same concentration of compound as the external environment at equilibrium. Since the encapsulation curtails at 1 hour (Figure 3c), the liposomes are assumed near equilibrated by then, with D:L ratios at $68 \%$ of the maximum equilibrium concentration after which it plateaued. We were interested in what might be causing the difference between predicted and observed D:L ratios, and what forces cause the association of DXR with the liposome membrane at temperatures below $T_{m}$. We hypothesized that the observed association between DXR and liposomes at temperatures $<T_{m}$ may be due to the interactions between the phosphate group in DSPC and $\mathrm{NH}_{2}$ group of DXR. Using molecular dynamic simulations, we investigated the molecular interactions at the atomic level.

Since the passage of DXR is largely mediated by diffusion, movement through the membrane is dictated by both the long range Van Der Waals (VdW) energy and short range electrostatic forces existing between 
DXR and lipid molecules ${ }^{38}$ (Figure $4 \mathbf{a}$ and $4 \mathbf{b}$, respectively). We explored the interactions, the $\mathrm{NH}_{2}$ group of DXR has with both the head and tail of the lipid. The analysis of VdW indicated that at $37^{\circ} \mathrm{C}$, the DXR molecule primarily interacts with the lipid head (average energy $-43.8 \mathrm{kcal} / \mathrm{mol}$ ), while interactions between DXR and the tails are negligible (average energy $-1.9 \mathrm{kcal} / \mathrm{mol}$ ). Similarly, the electrostatic interactions at the head (Figure $\mathbf{4 b}$ ) are highly attractive promoting the observed adsorption at temperatures lower than $T_{m}$ (Figure $3 b$ ). Conversely, at $T_{m}\left(55^{\circ} \mathrm{C}\right)$ when the lipid layer is more fluidic, the DXR first contacts the head (as indicated by the low VdW), but also moves closer to the tails (as evident by the decreasing VdW). At and above the $T_{m}$, as the DXR moves into the membrane, and thus further away from the charged phosphate heads, the attraction decreases ( $K d W$ becomes more positive), before returning to a more negative steady state. Interactions between the leaflet and the DXR molecule are illustrated in Figure 4c. Below $T_{m}$, DXR remains within the Van der Waals radii, interacting with the phosphate heads. However, as the membrane acquires a more liquid-state, the lipophilic region of DXR migrates into the tails. Most of the inter-atomic interactions occur within 5-8 Å of each other (Figure 4d), suggesting weaktransient interactions, with the majority occurring between DXR and the lipid head.

While temperature clearly plays a role in thermal equilibration of liposomes with their exterior, experimentally we observed an almost immediate deleterious response in liposome stability at temperatures greater than $T_{m}$. To elucidate this at the atomic level, we explored the effects of temperature on other factors that can influence DXR diffusion and membrane stabilization (Supplementary Figure 4a). In addition to the electrostatic and VdW interactions, the DXR molecule can stabilize via the additive effect of hydrogen bonding (Supplementary Figure $\mathbf{4 b}$ ). While the number of bonds initially starts equally, irrespective of temperature, there is a significant decrease as the temperature increases and as the DXR enters the membrane. Conversely, at lower temperatures, the hydrogen bonding steadily increases, thereby stabilizing the interactions. Interestingly, while there is a significant decrease in the average number of hydrogen bonds when increasing the temperature from 37 ${ }^{\circ} \mathrm{C}$ to $55^{\circ} \mathrm{C}(12.75$ vs 6.75 , respectively) there is very little difference between the number of hydrogen bonds at $55^{\circ} \mathrm{C}$ and $65^{\circ} \mathrm{C}$ (averages 6.75 and 4.5, respectively). This decrease in hydrogen bonding at temperatures $\geq T_{m}$ likely enhances the $D X R^{\prime} s$ ability to migrate into the tail section, however, the levels are re-established as the DXR progresses through the membrane, suggesting a rotation of the DXR molecule so that the $\mathrm{NH}_{2}$ group points towards the polar heads. These results are most evident in the RMSD of the DXR, where there is an increase in the average DXR movement of over $50 \%$ at $T_{m}$ compared to $37^{\circ} \mathrm{C}$, but only an $8 \%$ increase between $55^{\circ} \mathrm{C}$ and $65^{\circ} \mathrm{C}$ (Supplementary Figure 4c). Therefore, while there is a large difference in equilibration between $37^{\circ} \mathrm{C}$ and $55^{\circ} \mathrm{C}$, the change is not proportional above $\mathrm{T}_{\mathrm{m}}$.

The membrane fluidity that permits DXR infiltration, as well as the loss of stability that correlates with the observed liposome disintegration at higher temperatures, is seen most apparently in the conformational energy of the tails (Supplementary Figure 4d). While higher conformational energy (and thus membrane fluidity) will enhance DXR infiltration into the membrane ${ }^{39}$, our observations noted that liposomes rapidly destabilize at temperatures $>T_{m}$. Over the $18^{\circ} \mathrm{C}$ difference between gel phase $\left(37^{\circ} \mathrm{C}\right)$ and the $T_{m}\left(55^{\circ} \mathrm{C}\right)$, the conformational energy is increased $2.51 \%$. Whereas, a smaller $10^{\circ} \mathrm{C}$ change in the temperature from $55^{\circ} \mathrm{C}$ to $65^{\circ} \mathrm{C}$ netted a similar $2.52 \%$ increase in conformational energy. Taken together with our experimental results, we believe that this non-proportional increase in conformational energy results in the observed decrease in liposome stability. While liposomes can mitigate the initial increase in conformational energy, for at least our observation window ( 24 hours) (Figure $2 \mathbf{c}$ and $\mathbf{3 b}$ ), they are unable to remain stable with the additional increase in energy. Therefore, while increasing the temperatures 
beyond $T_{m}$ may increase DXR movement (Figure 4c), it also greatly decreases the membrane stability and thus does not offer a feasible approach to increase equilibration.

The Universal Nature of the Thermal Equilibration Approach

Many popular encapsulation approaches are drug specific and are thus not versatile. However, we foresee using passive equilibration as a universal encapsulation technique that could be applicable to a variety of molecules. To demonstrate this, we chose two small molecules that vastly vary in their polarity, Fluorescein, and Nile Red. These molecules are similar in size and have compatible excitation and emission spectra, which allows us to view the co-loading capabilities of our approach (Figure 5a). Fluorescein and Nile Red (solubilized in DMSO) were introduced to liposomal suspensions at concentrations of $750 \mathrm{mM}$ and $20 \mathrm{mM}$, respectively. Samples showed D: L levels of $0.03( \pm 0.013)$ and $0.003( \pm 0.000099)$, respectively (Figure 5b).

Furthermore, we demonstrate efficient targeting of liposomes to cancer cells and in vitro cellular uptake using the proposed technique (Figure 5c). Here, PEGylated liposomes were created through "Preinsertion" (DSPE-PEG-Mal was added into the IPA solution pre-synthesis), or "Post-insertion" (DSPE-PEGMal was integrated in following the synthesis via thermal equilibration along with fluorescein). Both approaches yielded functionalized targeting liposomes, as demonstrated by the presence of fluorescein when examining cells exposed to PEGylated liposomes, and comparing their fluorescence to cells introduced to non-PEGylated liposomes.

\section{Discussion}

We show here a universal approach to sequester small molecules within liposomes for drug delivery, irrespective of the drug polarity. We hypothesized that by incubating liposomes with small volumes of high concentration drug solution, the internal environment of liposomes would eventually equilibrate with the external environment, with localization largely depending on the partitioning coefficient of the compound (Figure 3a). We further hypothesized that this effect could be enhanced by increasing the fluidity of the membrane (via thermal incubation), which would also allow the introduction of targeting moieties within the membrane.

A lipid vesicle's $T_{m}$ is a physical characteristic that dictates the order of the lipid membrane at varying temperatures, and it is a crucial variable to consider in engineering liposomal delivery systems, where synthetic lipids have a higher $T_{m}$ than natural lipids ${ }^{40}$. DSPC is a synthetic, saturated, 18 carbon lipid with a $T_{m}$ of $55^{\circ} \mathrm{C}$. This elevated $T_{m}$ imparts several favorable characteristics on the liposome, including enhanced in vivo stability and reduced clearance rates compared to natural lipids ${ }^{41}$.

We synthesized liposomes via a modified form of our previously published SPIN method (Figure 1a) ${ }^{42}$, and concentrated using filter centrifugation, which we found was significantly superior to ultracentrifugation (Figure $\mathbf{1 b}$ and $1 \mathbf{c}$ ). Liposomes display high stability when incubated at the $\mathrm{T}_{\mathrm{m}}$ in up to $10 \%$ DMSO (as a vehicle of lipophilic molecules). We found that above this temperature, and when incubated with isopropyl or ethyl alcohol, liposomes rapidly fell apart resulting in the precipitation of lipids. However, previous reports by Balley et. al. have demonstrated that DSPC liposomes were stable for up to one hour at $70^{\circ} \mathrm{C}$ in up to $30 \%$ ethanol ${ }^{43}$. This discrepancy may be due to the increased levels of cholesterol (45\%) in their liposomes as compared to $33 \%$ cholesterol in our experiments, which is reported to be the most stable $41,44,45$. Cholesterol has a stabilizing effect, creating order in the hydrophobic region at increased 
temperatures that could stabilize liposomes at these increased temperatures ${ }^{46,47}$. In our simulations, we found no interaction between the DXR and cholesterol (Supplementary Figure 5), but simulations to determine the effect of increased cholesterol on conformational energy may explain this discrepancy. In our simulated leaflet containing 33\% cholesterol, we found little difference in the interactions between DXR and DSPC once the temperature was $>T_{m}$. Electrostatic, $V d W$, and hydrogen bonding interactions (Figures $4 \mathrm{a}$ and $\mathbf{4 b}$ ) were similar at both $55^{\circ} \mathrm{C}$ and $65^{\circ} \mathrm{C}$. However, there was a larger than expected increase in conformational energy when comparing $55^{\circ} \mathrm{C}$ and $65^{\circ} \mathrm{C}$ membrane simulations. This increase in conformational energy also affects other membrane dynamics which can lead to loss of stability, such as the solvent accessible surface area (Supplementary Figure 6).

We showed equilibration as a robust approach to create potent targeting liposomes. The liposomes quickly equilibrated within an hour (Figure 3b) and can provide a targeted therapeutic effect (Figure 3e). Interestingly, we found that even at lower temperatures (i.e. $<T_{m}$ ), DXR associates with liposomes. However, an increase in encapsulation was observed at higher temperatures, which is likely a direct result of the increased permeability of the membrane. The addition of cholesterol limits the phase transition of liposome membrane and maintains a liquid ordered phase ${ }^{48}$. Using NAMD to simulate the membrane dynamics and interatomic interactions, we investigated the association between liposomes and DXR at various temperatures. We found that the slight positive charge found on the amine group of DXR was highly attracted to the negative phosphate groups on lipids. This interaction elegantly explains the proficiency of active methods, wherein ion gradients (typically made from high intramolecular levels of sulphate and phosphate) attract slightly positive charged compounds such as DXR. The phosphate- $\mathrm{NH}_{2}$ attraction occurs through both long range ( $\mathrm{VdW})$ and intermediate range (electrostatic) interactions and allows for adsorption of DXR to the membrane. The adsorbed molecules are retained through all wash cycles but are quickly released once introduced to physiological conditions, as evidenced by the initial burst seen in Figure 3d. The concentration of DXR associated with liposomes decreases by $20 \%$ within the first two hours, but only $5 \%$ was released over the following 46 hours. This opens the exciting possibility of a tri-modal release, wherein liposomes can be engineered to contain DXR adsorbed to the liposome membrane (through low temperature equilibration) as well as encapsulated within the core (through passive encapsulation) and membrane (through high temperature equilibration).

In addition to drug compartmentalization, thermal equilibration also conveniently allows for the coloading of compounds. Liposomes are promising avenues of increasing therapeutic index through combinatorial simultaneous delivery ${ }^{49,50}$. Recently, researchers have investigated the use of liposomes coloaded with doxorubicin and paclitaxel, molecules that differ greatly in polarity ${ }^{51}$. Combined with our liposome synthesis and concentration strategy, we demonstrate here that the thermal equilibration approach effectively sequesters both hydrophilic and lipophilic compounds within targeting liposomes (Figure 5b) without requiring specialized steps. Furthermore, we can encapsulate both types of molecules at the same time, generating co-loaded targeting liposomes in a more efficient manner than passive encapsulation. While the goal of this work was to demonstrate that antibody coupling is possible during equilibration, future work characterizing the antibody insertion and efficiency (including maleimide hydrolysis) warrants further investigation. This will be highly variable depending on the intended application, and thus is not within the scope of the current work. Exploration on the compatibility of liposomes equilibrated in solvents other than DMSO, such as surfactants and emulsifiers, will greatly advance the use of equilibration in generating highly potent, co-loaded liposomes.

\section{Conclusions}


In this work, we describe a novel strategy for rapidly generating potent targeting liposomes using the thermal equilibration approach. We characterize the stability and encapsulation potential of liposomes both experimentally and through molecular dynamic simulations. We then demonstrate the therapeutic capability by introducing targeted LDXR to cancer cells in vitro and evaluating cell viability. Finally, we demonstrate the universal nature of this approach by co-loading both hydrophilic and lipophilic molecules. Future work will consist of drug specific optimizations using microscopy and further cellular uptake assays to establish potent delivery systems. We believe that thermal equilibration offers unparalleled advantages compared to conventional encapsulation techniques with a variety of potential applications in the therapeutic and theranostic landscape.

\section{Author Information}

\section{Conflicts of interest}

The authors have no conflicts to declare.

\section{Corresponding Author}

*111 Michigan Ave NW, Washington, DC 20010, USA. Email: nagrawal2@Childrensnational.org Telephone: 202-476-3072

Author Contributions

NA and SR conceived the technology and designed the research plan. SR and CL synthesized liposomes and performed characterization experiments and data analysis. SS performed membrane simulations. SR wrote the initial manuscript draft. All authors discussed the results and edited the manuscript. All authors assume responsibility for their respective contributions and the overall work presented in the manuscript.

\section{$\underline{\text { Funding Sources }}$}

This work was supported by funding from the National Science Foundation award\# 1645195 and 2013952, and Children's National Medical Center.

\section{$\underline{\text { Acknowledgments }}$}

The authors are grateful to Dr. Rohan Fernandes at The George Washington University for allowing the use of their analytical equipment and other resources for this project. 
a

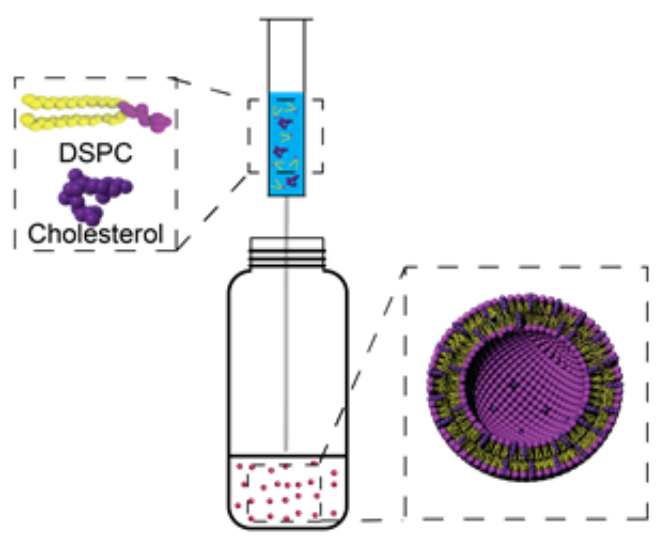

C

Liposomes Retained by Lipid Concentration

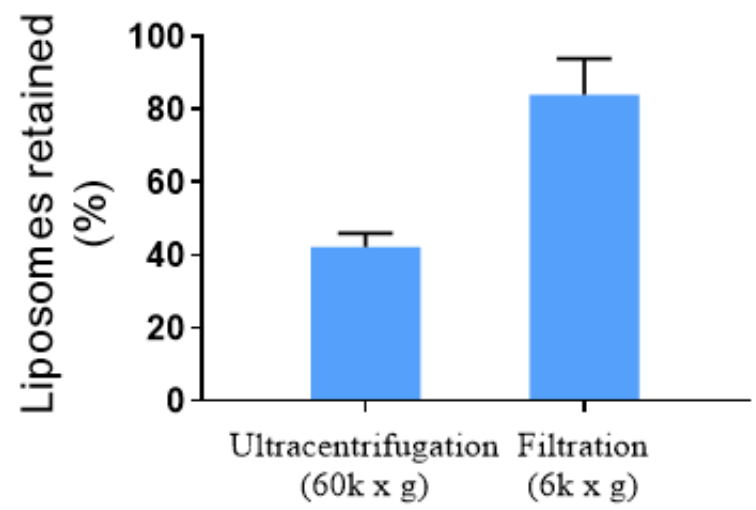

b Change in liposome size

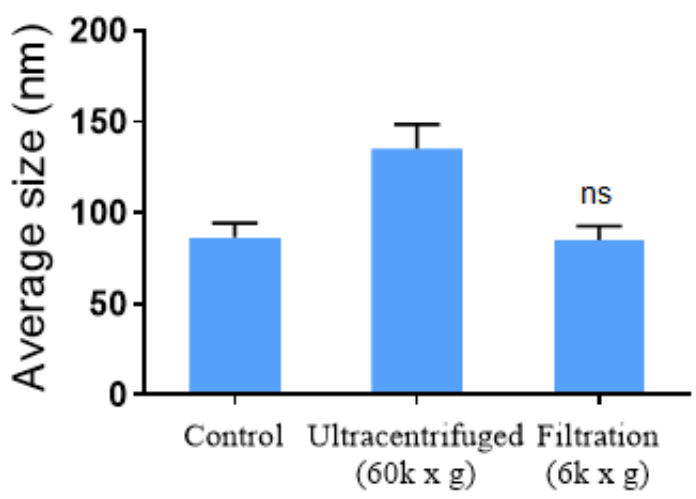

\section{Theoretical D:L ratio}

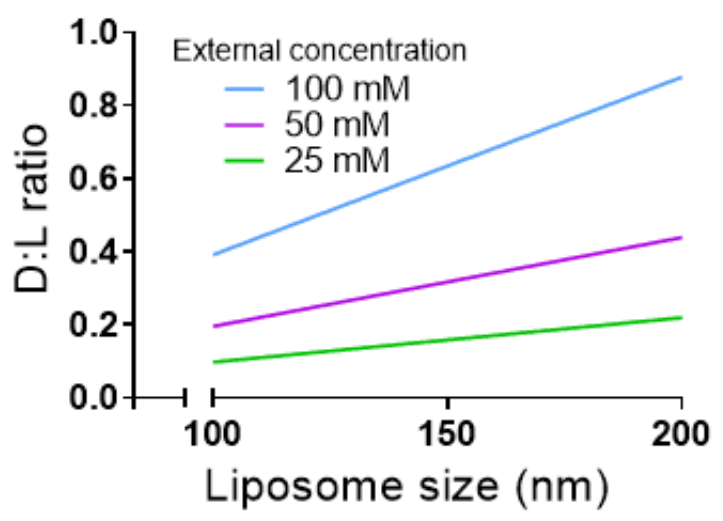

Figure 1: Synthesis of potent targeted liposomes. (a) Liposomes are synthesized in a rapid and scalable manner using the injection method. Membrane components are dissolved in isopropyl alcohol and injected into $\mathrm{DI}$ water at $55^{\circ} \mathrm{C}$. The phase change causes the arrangement of liposome components to form small unilamellar liposomes. The solution is concentrated $20 x$ using filter centrifugation. (b and $c$ ) The filtration process retains the population of liposomes efficiently, as seen in the size distribution and lipid concentration. The liposome population increases in diameter from $86.6 \mathrm{~nm}$ to $135.4 \mathrm{~nm}$ when ultracentrifuged as a result of small liposomes remaining in the supernatant. The change in diameter when filtered was not statistically significant, suggesting the relative population was retained. Analysis of lipid concentration showed nearly $60 \%$ of the liposomes were lost following ultracentrifugation, whereas filtration retained $84 \%$ of the total liposomes in solution. (d) The theoretical D:L ratio of thermal equilibration for hydrophilic molecules is dependent on liposome diameter and initial drug concentration, where increasing liposome diameter and external concentration results in an increase in liposome potency. 
a

\section{Effect of Additives}

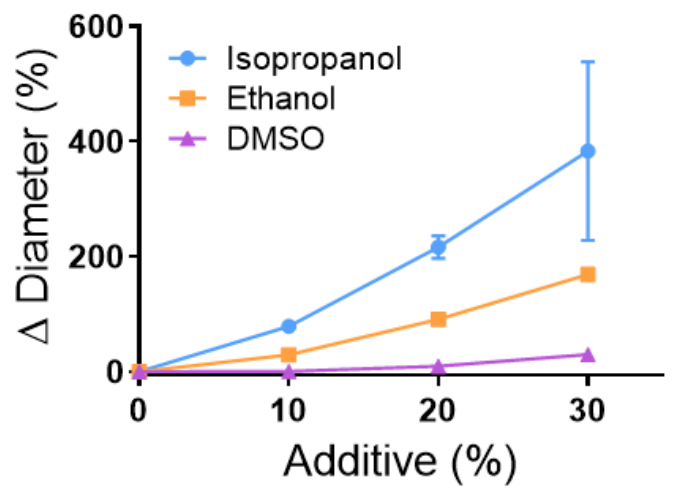

b

\section{Stability in DMSO for $24 \mathrm{~h}$}

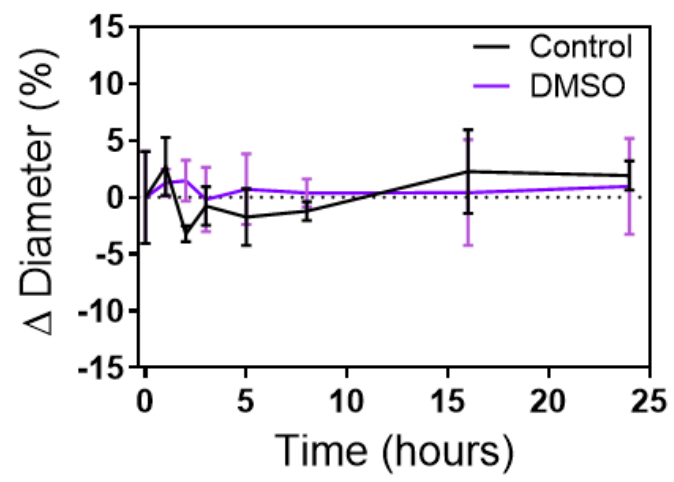

Figure 2: Analyzing the effect of temperature and solvents on liposome stability. (a) Liposomes incubated at $55^{\circ} \mathrm{C}$ for one hour with $10 \%$ DMSO did not show a statistically significant change in mean diameter $(p=0.9565)$. However higher levels of DMSO and all tested concentrations of IPA and EtOH drastically affected the population. (b) Liposomes incubated at $55^{\circ} \mathrm{C}$ with and without $10 \%$ DMSO were stable for up to 24 hours and showed no significant change in liposome diameter. 
a

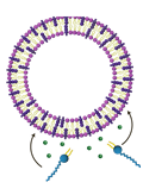

b

$$
\text { Effect of temperature }
$$
on equilibration

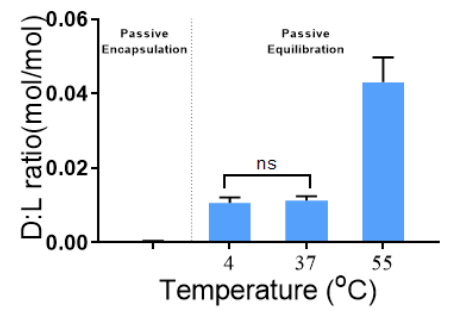

d

Drug release profile

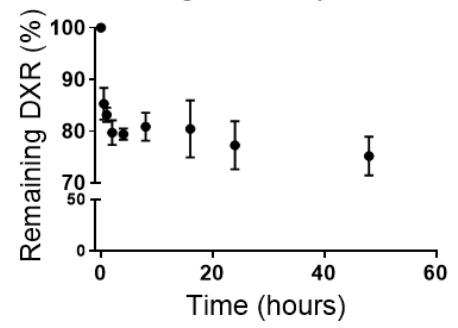

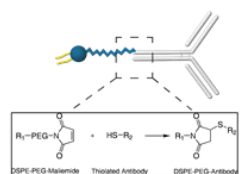

$\mathrm{C}$

Effect of incubation time on drug encapsulation

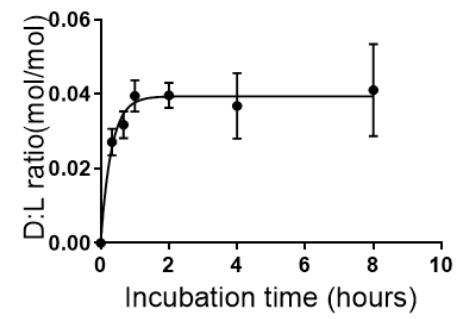

e

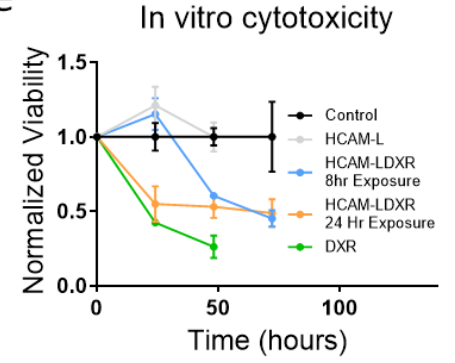

Figure 3: Thermal equilibration (a) Liposomes are equilibrated by incubating concentrated slurries with small molecules. Simultaneously, the liposomes can be incubated with DSPE-PEG-MAL linked to an antibody. This pegylated form of the potent liposome can be coupled to antibodies for efficient drug delivery. (b) The effect of temperature on equilibration was investigated by incubating DXR and liposomes at 4,37 , and $55^{\circ} \mathrm{C}$ for one hour. These results were compared to liposomal DXR created via passive encapsulation. $E_{T}$ increases $D: L$ nearly 200 folds compared to passive encapsulation, and even at low temperatures DXR is associated with the liposomes. (c) To optimize the encapsulation approach, liposomal samples were equilibrated for varying durations (up to 8 hours) with DXR. At indicated time intervals, liposomes were purified and the amount of DXR entrapped was measured through spectrometry. The liposomes showed a time dependent increase in DXR encapsulation up to 1 hour, however beyond 1 hour there was very little change. (d) Evaluations of the release of DXR were conducted by incubating $E_{T}$ liposomal DXR at $37^{\circ} \mathrm{C}$ in PBS with $10 \%$ FBS. The results demonstrate that there is a controlled escape of DXR from liposomes, with 75\% ( $\pm 37 \%$ ) of the DXR remaining in the liposomes after 48 hours. (e) During equilibration, DSPE-PEG-MAL can be integrated within the membrane, which when incubated with an antibody (HCAM), generates potent targeted liposomes. These targeted liposomes were cytotoxic when introduced to cancer cells in vitro, with a $52 \%$ decrease in cancer cell viability following 3 days of incubation. All measurements on the encapsulated DXR concentrations were determined using spectrometry and comparing absorbance values to a standard curve of known concentrations. Data was taken on at least 3 independently produced samples $(n=3)$ and measurements on each sample were made in triplicate. 


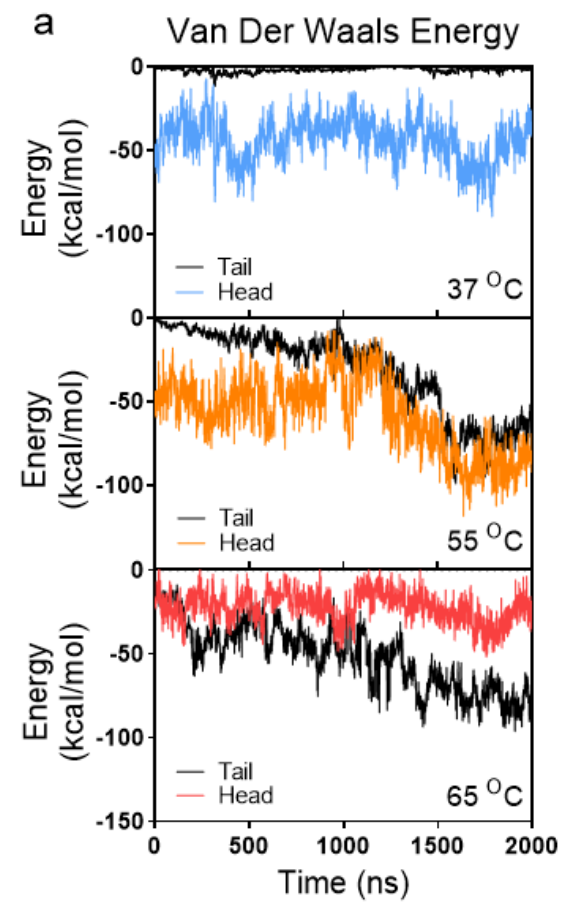

C

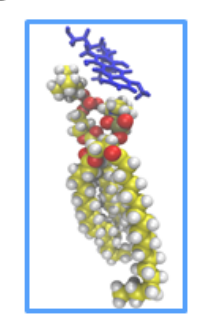

b
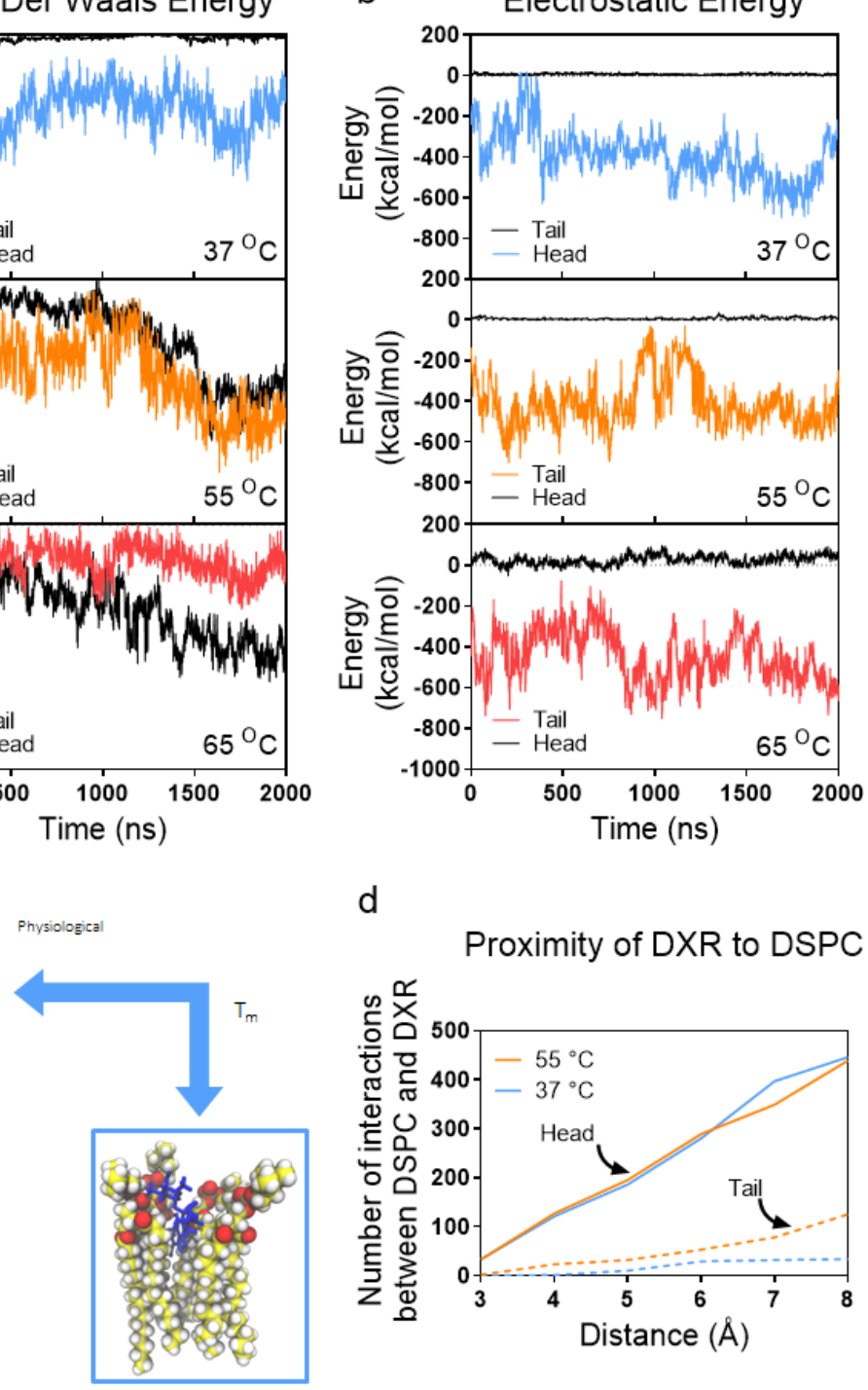

d

Proximity of DXR to DSPC

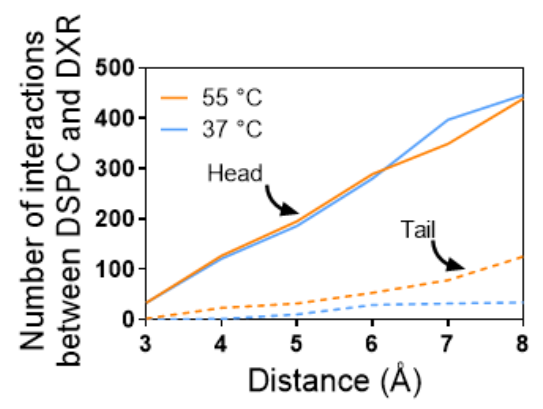

Figure 4: Atomic level simulations. Analysis of (a) long-range VdW and (b) short range electrostatic forces. At all temperatures, the DXR molecules are attracted to the negatively charged lipid heads, however only as the membrane acquires a more liquid-state $(\geq T \mathrm{~m})$ does the DXR shows any attraction or interaction to the lipid tails. (c) The simulations depict an adsorption of DXR to the membrane below $T_{m}$, however at and above $T_{m}$ DXR infiltrates into the membrane. (d) Most DXR atoms are located within 5-8 $\AA$ of DSPC, insinuating a more additive and transient non-covalent bond. 
a

\section{Dye Compatability}

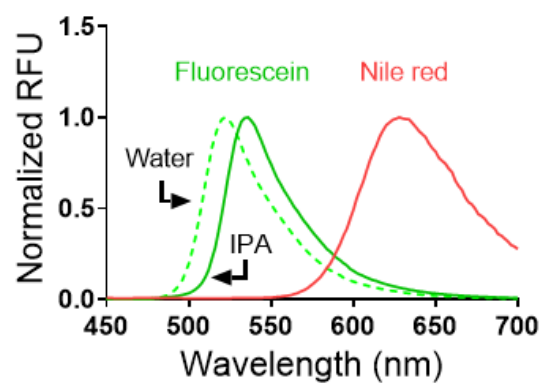

b

Coloaded Liposome Potency

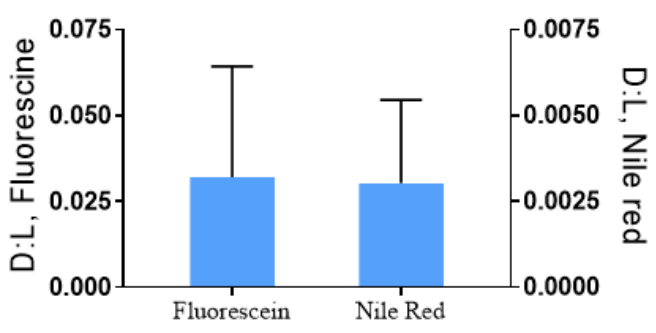

DAPI

Overlay
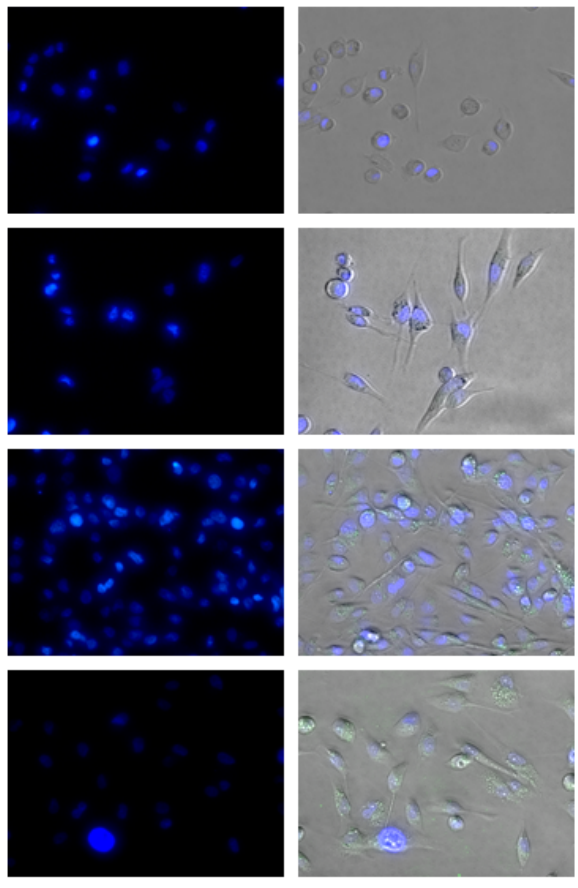

Figure 5: The use of thermal equilibration as a universal encapsulation method. Liposomes are uniquely capable of sequestering both lipophilic and hydrophilic molecules. (a) Using the equilibration approach, liposomes were loaded with the hydrophilic and lipophilic dyes Fluorescein and Nile Red, respectively. These dyes differ greatly in fluorescence emission and can be easily distinguished. (b) The liposomes were then co-loaded by incubating with a mix of fluorescein and Nile Red. Analysis of liposome emission spectra reveal two peaks indicative of both dyes being sequestered. (c) Liposomes loaded with fluorescein (green) were targeted to MDA-MB-231 breast cancer cells in vitro using PEG-DSPE-Mal covalently bonded to CD44 as a targeting molecule. The PEGylated lipid was introduced either during vesiculation or during thermal equilibration. 


\section{References}

1 Fendler, J. H. \& Romero, A. Liposomes as drug carriers. Life Sciences 20, 1109-1120, doi:10.1016/0024-3205(77)90481-7 (1977).

2 Tiwari, G. et al. Drug delivery systems: An updated review. International Journal of Pharmaceutical Investigation 2, 2-11, doi:10.4103/2230-973X.96920 (2012).

3 Gregoriadis, G. Liposomes in Drug Delivery: How It All Happened. Pharmaceutics 8, 19, doi:10.3390/pharmaceutics8020019(2016).

4 Svenson, S. Theranostics: Are We There Yet? Molecular Pharmaceutics 10, 848-856, doi:10.1021/mp300644n (2013).

5 Xing, H., Hwang, K. \& Lu, Y. Recent Developments of Liposomes as Nanocarriers for Theranostic Applications. Theranostics 6, 1336-1352, doi:10.7150/thno.15464 (2016).

6 Sachin, M., Sachin, D. \& Dinesh, S. Voyage of theranostic liposomes for imaging and therapy. Journal of Cosmetic and Laser Therapy 19, 245-249, doi:10.1080/14764172.2017.1279331 (2017).

7 Van Slooten, M. L. et al. Liposomes as sustained release system for human interferon-Q: biopharmaceutical as pects. Biochimica et Biophysica Acta 1530, 145 (2001).

$8 \quad$ Zylberberg, C. \& Matosevic, S. Pharmaceutical liposomal drug delivery: a review of new delivery systems and a look at the regulatory landscape. Drug Delivery 23, 3319-3329, doi:10.1080/10717544.2016.1177136(2016).

9 Barenholz, Y. Doxil ${ }^{\circledR}$ - The first FDA-approved nano-drug: Lessons learned. Journal of Controlled Release 160, 117-134, doi:10.1016/j.jconrel.2012.03.020(2012).

10 Linden, P. K. Amphotericin B lipid complex for the treatment of invasive fungal infections. Expert Opinion on Pharmacotherapy 4, 2099-2110, doi:10.1517/14656566.4.11.2099 (2003).

11 Peravali, R. et al. Enhancing the Enhanced Recovery Program in Colorectal Surgery - Use of Extended-Release Epidural Morphine (DepoDur ${ }^{\circledR}$ ). Annals of Coloproctology 30, 186-191, doi:10.3393/ac.2014.30.4.186(2014).

12 Park, K. S., Sun, X., Aikins, M. E. \& Moon, J. J. Non-viral COVID-19 vaccine delivery systems. Adv Drug Deliv Rev 169, 137-151, doi:10.1016/j.addr.2020.12.008 (2021).

13 Jaafar-Maalej, C., Diab, R., Andrieu, V., Elaissari, A. \& Fessi, H. Ethanol injection method for hydrophilic and lipophilic drug-loaded liposome preparation. Journal of Liposome Research 20, 228-243, doi:10.3109/08982100903347923 (2010).

14 Gubernator, J. Active methods of drug loading into liposomes: recent strategies for stable drug entrapment and increased <i>in vivo</i> activity. Expert Opinion on Drug Delivery 8, 565-580, doi:10.1517/17425247.2011.566552 (2011).

15 Dua, J. S., Rana, A. C. \& Bhandari, A. K. Liposome: methods of preparation and applications. Int J Pharm Stud Res 3, 14-20 (2012).

16 Wang, J. P., Maitani, Y., Takayama, K. \& Nagai, T. Pharmacokinetics and antitumor effect of doxorubicin carried by stealth and remote loading proliposome. Pharmaceutical Research 17, 782-787 (2000).

17 Fritze, A., Hens, F., Kimpfler, A., Schubert, R. \& Peschka-Süss, R. Remote loading of doxorubicin into liposomes driven by a transmembrane phosphate gradient. Biochimica et Biophysica Acta (BBA)- Biomembranes 1758, 1633-1640, doi:10.1016/j. bbamem.2006.05.028 (2006).

18 Sur, S., Fries, A. C., Kinzler, K. W., Zhou, S. \& Vogelstein, B. Remote loading of preencapsulated drugs into stealth liposomes. Proceedings of the National Academy of Sciences 111, 2283-2288, doi:10.1073/pnas.1324135111 (2014).

19 Roberts, S. A., Parikh, N., Blower, R. J. \& Agrawal, N. SPIN: Rapid Synthesis, Purification, and Concentration of Small Drug Loaded Liposomes. Journal of Liposome Research, 1-30, doi:10.1080/08982104.2017.1381115(2017). 
Moser, M. et al. Quantification of PEG-Maleimide Ligands and Coupling Efficiencies on Nanoparticles with Ellman's Reagent. Analytical Chemistry 87, 9376-9383, doi:10.1021/acs.analchem.5b02173 (2015).

21 Stewart, J. C. Colorimetric determination of phospholipids with ammonium ferrothiocyanate. Analytical Biochemistry 104, 10-14 (1980).

22 Fang, X. J.et al. Doxorubicin induces drug resistance and expression of the novel CD44st via NFKB in human breast cancer MCF-7 cells. Oncology Reports 31, 2735-2742 (2014).

23 Brooks, B. R. et al. CHARMM: The Biomolecular Simulation Program. Journal of computational chemistry 30, 1545-1614, doi:10.1002/jcc.21287 (2009).

24 Akbarzadeh, A. et al. Liposome: classification, preparation, and applications. Nanoscale Res Lett 8, 102 (2013).

25 Zhang, H. in Liposomes Vol. 1522 (eds Gerard G. M. D'Souza \& Gerard G. M. D'Souza) 17-22 (2017).

26 Lapinski, M. M., Castro-Forero, A., Greiner, A. J., Ofoli, R. Y. \& Blanchard, G. J. Comparison of Liposomes Formed by Sonication and Extrusion: Rotational and Translational Diffusion of an Embedded Chromophore. Langmuir 23, 11677-11683, doi:10.1021/la7020963 (2007). Ong, S. G. M., Chitneni, M., Lee, K. S., Ming, L. C. \& Yuen, K. H. Evaluation of Extrusion Technique for Nanosizing Liposomes. Pharmaceutics 8, doi:10.3390/pharmaceutics8040036 (2016).

28 Traïkia, M., Warschawski, D. E., Recouvreur, M., Cartaud, J. \& Devaux, P. F. Formation of unilamellar vesicles by repetitive freeze-thaw cycles: characterization by electron microscopy and 31P-nuclear magnetic resonance. European biophysics journal: EBJ 29, 184-195 (2000).

29 López-Pinto, J. M., González-Rodríguez, M. L. \& Rabasco, A. M. Effect of cholesterol and ethanol on dermal delivery from DPPC liposomes. International Journal of Pharmaceutics 298, 1-12, doi:10.1016/j.ijpharm.2005.02.021 (2005).

30 Roberts, S. A., Neelaveni, N. \& Agrawal, N. 64-67 (2017).

31 Alyane, M., Barratt, G. \& Lahouel, M. Remote loading of doxorubicin into liposomes by transmembrane $\mathrm{pH}$ gradient to reduce toxicity toward H9c2 cells. Saudi Pharmaceutical Journal 24, 165-175, doi:10.1016/j.jsps.2015.02.014 (2016).

32 Ishida, O., Maruyama, K., Sasaki, K. \& Iwatsuru, M. Size-dependent extravasation and interstitial localization of polyethyleneglycol liposomes in solid tumor-bearing mice. International Journal of Pharmaceutics 190, 49-56 (1999).

33 Drummond, D. C., Noble, C. O., Hayes, M. E., Park, J. W. \& Kirpotin, D. B. Pharmacokinetics and in vivo drug release rates in liposomal nanocarrier development. Journal of Pharmaceutical Sciences 97, 4696-4740, doi:10.1002/jps.21358 (2008).

34 Fugit, K. D. et al. Mechanistic model and analysis of doxorubicin release from liposomal formulations. Journal of Controlled Release 217, 82-91, doi:10.1016/j.jconrel.2015.08.024 (2015).

35 Dos Santos, N. et al. Influence of poly(ethylene glycol) grafting density and polymer length on liposomes: Relating plasma circulation lifetimes to protein binding. Biochimica et Biophysica Acta (BBA) - Biomembranes 1768, 1367-1377, doi:10.1016/j.bbamem.2006.12.013 (2007).

36 Huang, Z. \& King, M. R. An immobilized nanoparticle-based platform for efficient gene knockdown of targeted cells in the circulation. Gene therapy 16, 1271-1282 (2009).

37 Olsson, E. et al. CD44 is oforms are heterogeneously expressed in breast cancer and correlate with tumor subtypes and cancer stem cell markers. BMC Cancer 11, 418, doi:10.1186/1471-2407-11418 (2011).

38 Raschke, T. M., Tsai, J. \& Levitt, M. Quantification of the hydrophobic interaction by simulations of the aggregation of small hydrophobic solutes in water. Proceedings of the National Academy of Sciences 98, 5965-5969, doi:10.1073/pnas.111158498 (2001). 
39 Sharma, V. K., Sarwa, K. K. \& Mazumder, B. Fluidity enhancement: a critical factor for performance of liposomal transdermal drug delivery system. Journal of Liposome Research 24, 83-89, doi:10.3109/08982104.2013.847956(2014).

$40 \mathrm{Li}$, J. et al. A review on phospholipids and their main applications in drug delivery systems. Asian Journal of Pharmaceutical Sciences 10, 81-98, doi:10.1016/j.ajps.2014.09.004 (2015).

41 Allen, T. M., Hansen, C. \& Rutledge, J. Liposomes with prolonged circulation times: factors affecting uptake by reticuloendothelial and other tissues. Biochimica et Biophysica Acta (BBA) Biomembranes 981, 27-35, doi:10.1016/0005-2736(89)90078-3 (1989).

42 Roberts, S. A., Parikh, N., Blower, R. J. \& Agrawal, N. SPIN: rapid synthesis, purification, and concentration of small drug-loaded liposomes. Journal of Liposome Research 28, 331-340, doi:10.1080/08982104.2017.1381115 (2018).

43 Wehbe, M. et al. A simple passive equilibration method for loading carboplatin into pre-formed liposomes incubated with ethanol as a temperature dependent permeability enhancer. Journal of Controlled Release 252, 50-61, doi:10.1016/j.jconrel.2017.03.010 (2017).

44 Forssen, E. \& Willis, M. Ligand-targeted liposomes. Adv Drug Deliv Rev 29, 249-271, doi:10.1016/S0169-409X(97)00083-5 (1998).

45 Briuglia, M.-L., Rotella, C., McFarlane, A. \& Lamprou, D. A. Influence of cholesterol on liposome stability and on in vitro drug release. Drug Delivery and Translational Research 5, 231-242, doi:10.1007/s13346-015-0220-8 (2015).

46 Redondo-Morata, L., Giannotti, M. I. \& Sanz, F. Influence of Cholesterol on the Phase Transition of Lipid Bilayers: A Temperature-Controlled Force Spectroscopy Study. Langmuir 28, 1285112860, doi:10.1021/la302620t (2012).

47 Sadeghi, N. et al. Influence of cholesterol inclusion on the doxorubicin release characteristics of lysolipid-based thermosensitive liposomes. International Journal of Pharmaceutics, doi:10.1016/j.jpharm.2017.11.002 (2017).

48 Marsh, D. Liquid-ordered phases induced by cholesterol: a compendium of binary phase diagrams. Biochimica Et Biophysica Acta 1798, 688-699, doi:10.1016/j.bbamem.2009.12.027 (2010).

49 Shaikh, I. M. et al. Liposome co-encapsulation of synergistic combination of irinotecan and doxorubicin for the treatment of intraperitoneally grown ovarian tumor xenograft. Journal of Controlled Release: Official Journal of the Controlled Release Society, doi:10.1016/j.jconrel.2013.10.025 (2013).

50 Eloy, J. O. et al. Co-loaded paclitaxel/rapamycin liposomes: Development, characterization and in vitro and in vivo evaluation for breast cancer therapy. Colloids and surfaces. B, Biointerfaces 141, 74-82, doi:10.1016/j.colsurfb.2016.01.032 (2016).

51 Wang, Y. et al. Lung cancer combination therapy: co-delivery of paclitaxel and doxorubicin by nanostructured lipid carriers for synergistic effect. Drug Delivery 23, 1398-1403, doi:10.3109/10717544.2015.1055619(2016). 